\title{
Quantitative elastography of Achilles tendon using Shear Wave Elastography (SWE): correlation with zonal anatomy
}

\author{
François Petitpierre \\ Jean-Thomas Perez \\ Sylvain Bise \\ Claire Fournier \\ Olivier Hauger \\ Benjamin Dallaudière
}

MSK Imaging Department, Hôpital Pellegrin, CHU de Bordeaux, Bordeaux, France

Corresponding author:

Benjamin Dallaudiere

MSK Imaging Department

Hôpital Pellegrin, CHU de Bordeaux

1 place Amelie Raba Leon

33076 Bordeaux, France

E-mail: benjamin.dallaudiere@gmail.com

\section{Summary}

Introduction: To evaluate the feasibility and reproducibility of quantitative ultrasonic measurement of normal Achilles tendon (AT) stiffness, using supersonic shear wave elastography (SWE) in correlation with its zonal anatomy.

Materials and methods: Thirty tendons (8 women, 7 men) (mean age, 29,5 years; age range, 24-51 years) underwent morphologic and SWE measurements. The quantitative measurements of the tendon in four points (musculo-tendinous junction, tendinous body, pre-insertional area and enthesis) according to zonal anatomy were performed by two MSK radiologists and respectively expressed in terms of $\mathrm{mm}$ and Young's modulus (kPa). Inter-observer reproducibility was also assessed.

Results: The SWE measures show higher stiffness for the sagittal position of the US than in axial plane in all four AT levels $(p<0.0382)$. Moreover, SWE measurements were significantly higher in dorsal flexion than in plantar flexion in all four AT levels $(p<0.0317)$. Last, SWE visco-elasticity increases significantly from the musculotendinous junction to the pre-insertional and enthesis area $(p<0.001)$ in plantar flexion. There was no significant interobsever difference for morphologic measurements and for stifness measure- ments in plantar and dorsal flexions ( $p>0.075)$. Conclusion: Quantitative measurement of normal AT using SWE is reproductible almost in plantar flexion, and increase from musculo-tendinous junction to enthesis and could now be integrated in our routine AT ultrasound exploration. Level of evidence: II.

KEY WORDS: achilles, tendon, elastography, shearwave-elastography (SWE), ultrasound.

\section{Introduction}

Achilles tendinopathy has recently risen because of the over-use of this tendon in recreational but also sporting activities, in intermediate sport level and athletes population ${ }^{1,2}$. Beside the necessary load X-rays, ultrasonography (US) is the first examination to explore this anatomical structure because of the superficiality of the Achilles tendon (AT) and the availability of this technique.

Nowadays, the US exploration is usually based on the B-mode and Power Doppler-mode to assess tendon lesions, with better sensitivity but lower specificity than $\mathrm{MRI}^{3}$. Indeed, US is the indirect reflection of the healthiness of the tendon through evaluation of peri-tendinopathy, tendinopathy, tear, hyperhemia or calcification. This modality provides an anatomic precise imaging. However, it looks sometimes difficult to correlate tissue disorganization and clinical pain, especially in very previous symptoms. To go further in US assessment, elastography appeared and tried to provide functional characteristics of the stiffness behavior of the tendon. On the top of that, elastography software are more and more present on US system in clinical practice ${ }^{4,5}$.

Three main different elastography techniques have been developed. First, the strain elastography (SE) needs external manual compression from the operator which is efficient for superficial organs or used the compression generate by internal physiologic motion and permits a qualitative assessment ${ }^{6}$. Second, Acoustic Radiation Force Impulse (ARFI) strain imaging is based on the tissues displacements perpendicular to the surface by a short duration high intensity acoustic pushing pulse and permits quantitative assessment ${ }^{7,8}$. Last, the most recent technic is called Shear Wave Elastrography (SWE) and is based on the creation of dynamic stress (with 2 pushing pulse) 
to create shear waves into the tissue and their measurement ${ }^{9,10}$. Since 2012 , to our best knowledge, there have been many studies (around 45 publications) so far evaluating AT elastography, and discordant results have been reported. Kind of qualitative or quantitative data in elastography, in different ankle positions, in not the same clinical conditions, make the results difficult to interpret and then compare. Among them, 21 focused about healthy tendons and showed that elastography is feasible and reproductive in healthy volunteers ${ }^{11-14}$. Twelve studies used SWE with same conclusion on feasibility in normal AT, with a softer pathologic AT and lower shear wave speed in case of tendinopathy ${ }^{15}$.

Moreover, to our knowledge, except one cadaveric series $^{16}$, all these SWE series considerate AT as one unique entity and didn't takes in consideration AT anatomic regions and then biomechanical function and histology ${ }^{17}$.

The aim of this publication was 1 ) to assess the stiffness properties of the 4 different regions of normal AT by using SWE, in plantar and dorsal flexion; 2) to study its interobserver reproducibility and 3) try to propose an optimal anatomical and functional US protocol (within a reasonable time) in clinical practice.

\section{Materials and methods}

\section{Study Population}

Our study was submitted to the local institutional review board (CPP: Comité de Protection des Personnes du Sud-Ouest et d'Outre-Mer). None of the Authors received any support from any industry. All patients were volunteers and gave their written permission for anonymized use of their medical data for scientific purposes before the imaging examinations. They were prospectively included between February 2016 and May 2016.

The inclusion criteria for volunteers were age of 18 years or older. The exclusion criteria for volunteers were pregnancy, history of Achilles tendinopathy or AT surgery, pain in the AT. Volunteers with a history of systemic, metabolic, or endocrine diseases, psoriasis and those received corticosteroids, estrogens, quinolones, and cholesterol drugs were excluded because of known associations between those factors and tendinopathy ${ }^{18,19}$. Volunteers with a VISA-A score $<90$, were also excluded because of high risk of Achilles tendinopathy ${ }^{20}$.

The age of AT was calculated by using the difference between inclusion date and birthdate.

\section{US Procedure}

All examinations were performed bilaterally in the same time procedure to a standardized protocol (descripted below) with the same US system (SuperSonic Imagine ${ }^{\circledR}$, Aix-en-Provence, France) equipped with a 4-15 MHz superficial linear transducer. All measurements were realized by 2 (Musculo-skeletal)
MSK radiologists (respectively with 3 and 6 years post residency experience), at the same room temperature $\left(20^{\circ} \mathrm{C}\right)$, the same day for each patient.

The tendon was divided in 4 anatomical regions: musculo-tendinous junction, body area, pre-insertional area (1 $\mathrm{cm}$ upwards the enthesis) and enthesis ${ }^{15}$. First, pathologic AT characteristics were searched: enlargement (tendon thickness $>5$ millimeters) ${ }^{21}$, tendinosis, tear, calcification or hyperemia. Second, morphologic measurements were realized in dorsal flexion with the evaluation of the tendon thickness at the 4 AT levels described above and expressed in millimeters $(\mathrm{mm})$.

Third, SWE quantitative measurement were obtained by placing a $1 \mathrm{~mm}$ diameter $\mathrm{ROI}$ in the tendon, on the most homogenous area of the elastogram, determined by the operator over the color qualitative cartography of the AT stiffness. One ROI was placed on the axial slice at each AT level, in each 4 quarter of the slide (supero-medial, supero-lateral, infero-medial, infero-lateral). Then a ROI was placed on the sagittal medial and lateral slice, also in the 4 AT portions (Figs. 1, 2).

No gel pad was used but a minimum $5 \mathrm{~mm}$ thickness of US gel (measured on the B mode) was necessary in order to make SWE measurements, to compensate the ankle curve with the linear probe ${ }^{22}$.

All measurements were realized in dorsal flexion with tiptoes on the examination bed (ankle at $90^{\circ}$, Fig. 3A) and repeated in plantar flexion with the back of the foot on the examination bed (ankle at $160^{\circ}$, Fig. 3B).

The mean and standard deviation (SD) of stiffness in kilo-Pascal (kPA) were recorded for each ROI.

\section{Statistical analysis}

The software SAS (SAS for Windows version 9.3; SAS Institute, Cary, NC) was used to support the data sets.

Continuous variables were expressed as mean and $\mathrm{SD}$ in millimeters for morphologic assessment and in $\mathrm{kPa}$ for stiffness values.

SWE sagittal and axial variables were compared between the 4 different AT regions, in plantar and dorsal AT flexion, for the 2 MSK radiologists, using Student $t$ test. Interobserver reproducibility was also assessed. An overall $P$ value of less than 0.05 was considered to indicate a significant difference.

\section{Results}

Population and morphologic US measurements Fifteen healthy volunteers (i.e., 30 normal ATs) were included. Mean age was 29,5 years old (SD: 6,93). There were 7 males (47\%) and 8 females (53\%). There were as many left tendons as right because all subjects were analyzed both sides.

First, no pathologic AT characteristics were founded. No significant difference was noticed between all AT morphologic measurements between the 2 readers $(p>0,42)$. 


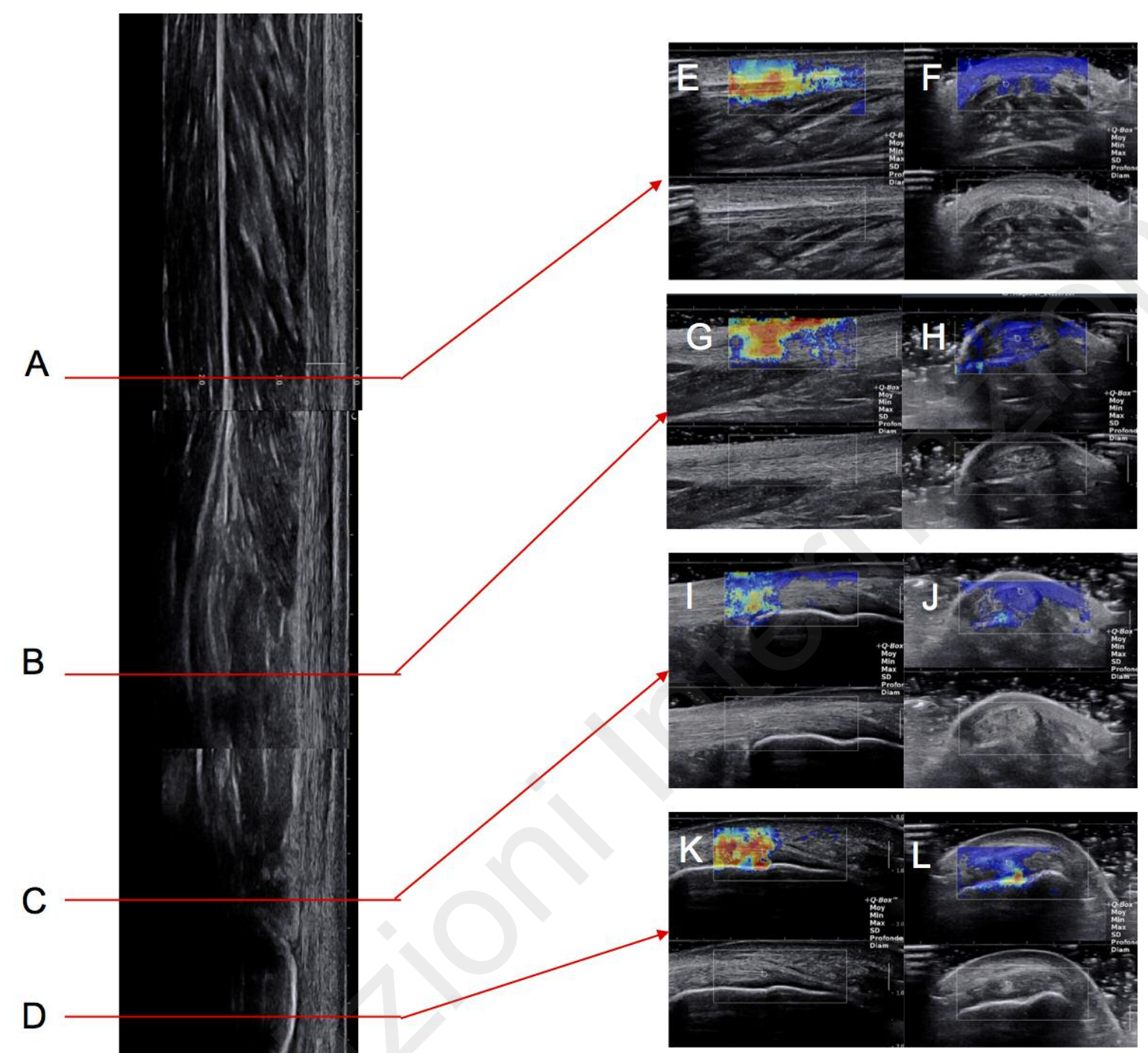

Figure 1. Panoramic reconstruction sonographic sagittal view of the AT in dorsal flexion with correspondent SWE acquisition of the 4 regions in sagittal and axial plane.

Table I resumed the morphologic antero-posterior diameter (in $\mathrm{mm}$ ) of the 30 studied AT for both operators.

\section{SWE US measurements}

The measurements show higher stiffness for the sagittal position of the US than in axial plane in all four AT levels $(p<0.0382)$. Moreover, SWE measurements were significantly higher in dorsal flexion than in plantar flexion in all four AT levels $(p<0.0317)$.

Last, SWE stiffness increases significantly from the musculo-tendinous junction to the pre-insertional and enthesis area $(p<0.001)$ in plantar flexion ankle position.

These results were independent of the AT side: indeed, there was no significant difference according to the AT side ( $p>0.8)$.

Table II resumed the stiffness (in kPA) of the 4 different portions of the tendon in axial and sagittal for both operators, in dorsal and plantar position.

\section{Interobserver reproducibility}

There was no significant difference between the two radiologists for all the measurements in plantar and dorsal flexions ( $p>0.075)$.

Table III resumed the inter observer reliability of SWE stiffness of the 4 different portions of the tendon in axial and sagittal for both operators and both side, in dorsal and plantar position.

According to Table III, perfect interobserver correlation was obtained for the plantar flexion and lower interobserver reproducibility was obtained for the dorsal flexion, especially for the enthesis region.

\section{Discussion}

Our study confirmed that AT stiffness is more important in sagittal plane than in axial plane and in dorsal flexion than in plantar flexion. Moreover, our results also showed that SWE stiffness increases significant- 


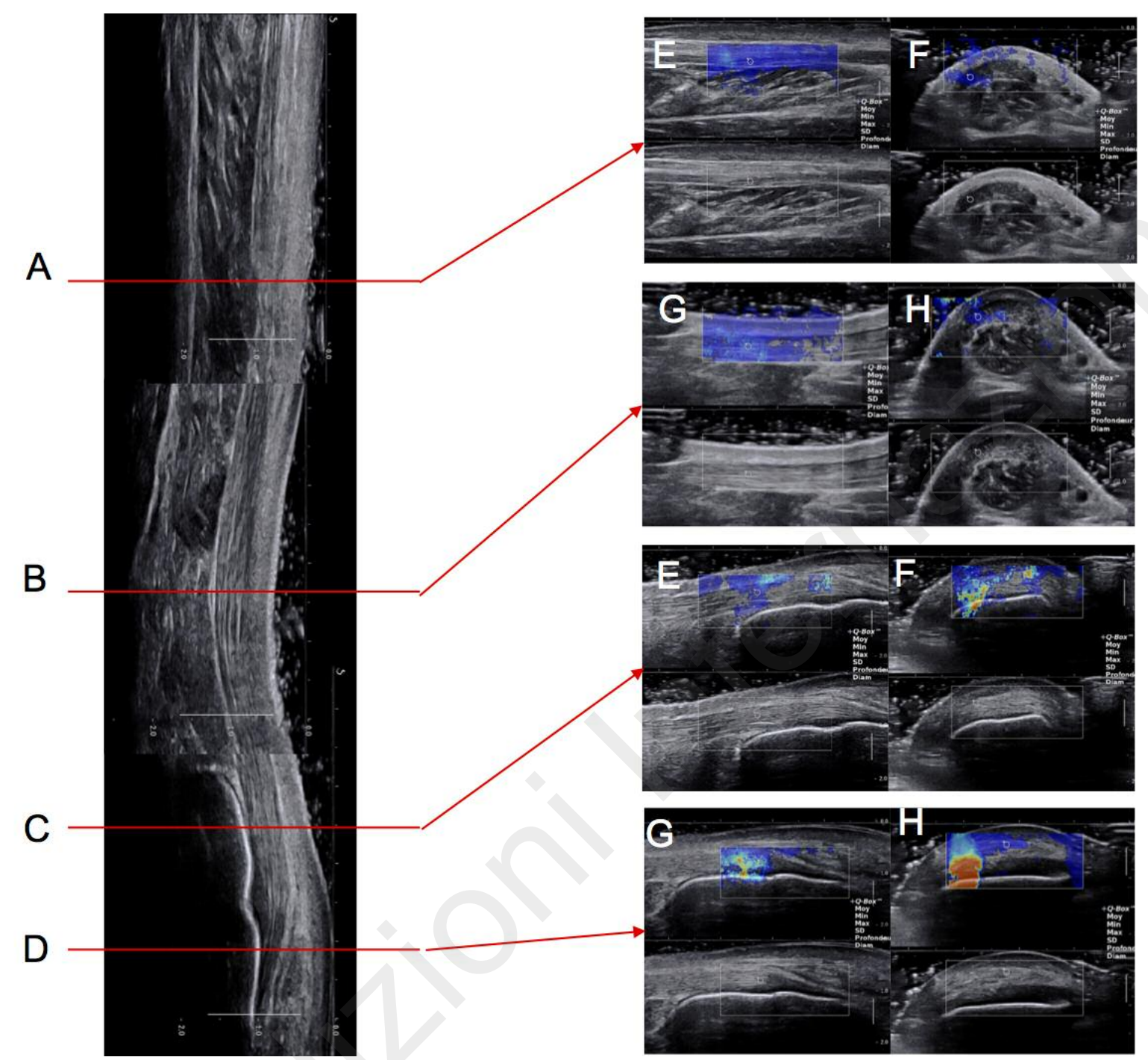

Figure 2. Panoramic reconstruction sonographic sagittal view of the AT in plantar flexion with correspondent SWE acquisition of the 4 regions in sagittal and axial plane.

Table I. Morphologic antero-posterior diameter $(\mathrm{mm})$ of the $\mathbf{3 0}$ studied Achilles tendons for the 2 readers. Mtj: Myo-tendinous junction.

\begin{tabular}{llll}
\hline & Operator $\mathbf{1}$ & Operator $\mathbf{2}$ & $\mathbf{p}$ \\
\hline Mtj & 1,77 & 1,65 & 0.4212 \\
Body & 4,00 & 4,01 & 0.5889 \\
Pre insertional & 3,61 & 3,39 & 0.5491 \\
Enthesis & 2,89 & 2,68 & 0.7043 \\
\hline
\end{tabular}

ly from the musculo-tendinous junction to the enthesis area in plantar flexion with a perfect interobserver correlation.

Our results are concordant with most part of recent SWE literature, despite controversial conclusions concerning elastography.
On one hand, in 2011, Aubry et al. studied the AT of 30 healthy subjects in 3 positions and demonstrated good intra individual correlation with interobserver reproducibility assessment in an unique measure on AT body ${ }^{12}$. Then, they explored healthy and pathologic tendon stiffness by SWE but with only one operator. This study showed a softening of the mid portion of the tendon for pathologic tendons, with a high specificity and low sensitivity. The mean stiffness was higher in the sagittal position than in the axial because the strong anisotropy of the tendon. Shear waves propagate slower perpendicularly to the tendon ${ }^{15}$. Our results are consistent with these series concerning AT healthy body assessment but show also the same difference between axial and sagittal measurements ${ }^{12,15}$.

In the same way, Suydam et al. studied 29 healthy volunteers and showed that there was no difference between right and left AT assessment ${ }^{23}$. One single 

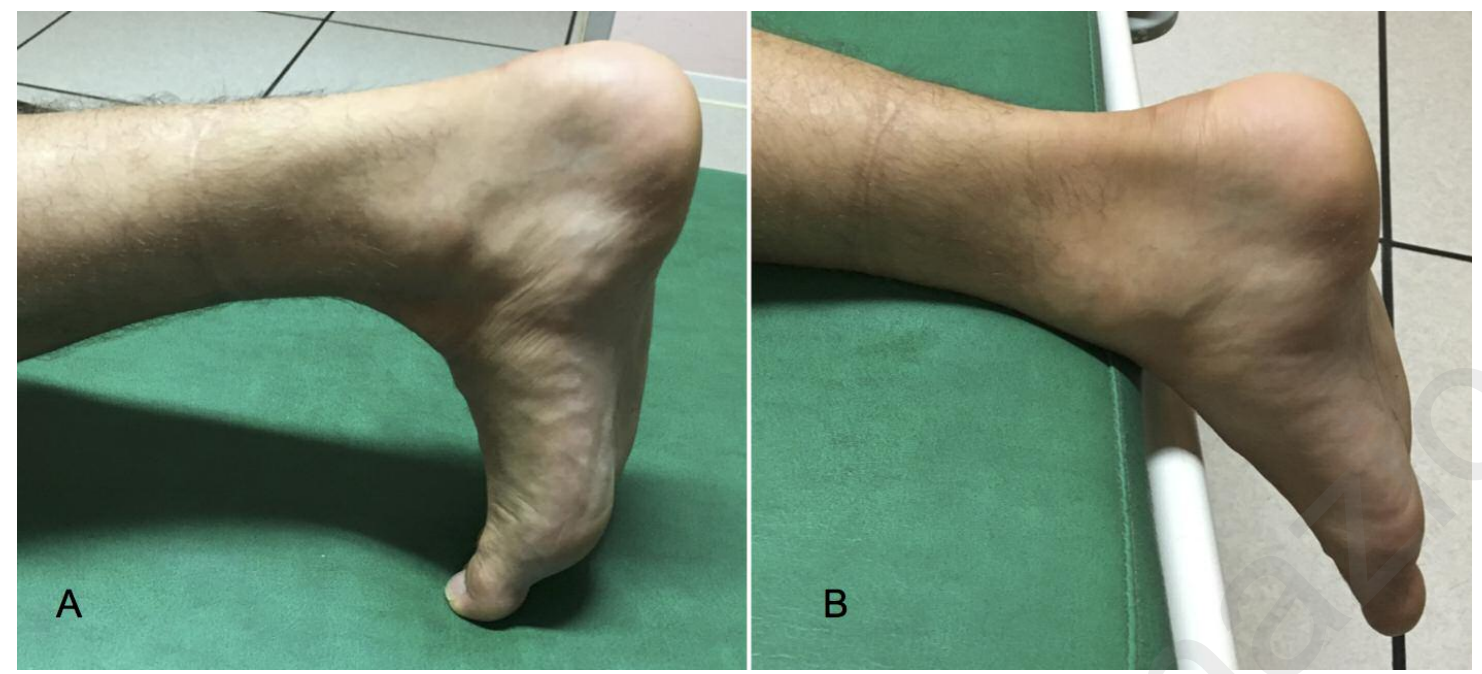

Figure 3. Picture of patient ankle position during examination with dorsal flexion (A) and plantar flexion (B).

Table II. SWE stiffness (kPA) of the 4 different portions of the Achilles tendon in axial and sagittal plane, for both operators (op1: operator1; op2: operator2), in dorsal and plantar position. Mtj: Myo-tendinous junction.

\begin{tabular}{|c|c|c|c|c|c|}
\hline & \multicolumn{5}{|c|}{ DORSAL FLEXION } \\
\hline & Axial & mean & SD & $\min$ & $\max$ \\
\hline \multirow[t]{4}{*}{ OP 1} & $\mathrm{Mtj}$ & 54,6 & 27,3 & 2,2 & 150,6 \\
\hline & body & 79,5 & 111,9 & 2,2 & 752 \\
\hline & pre insertional & 57,8 & 50,0 & 1,3 & 250,2 \\
\hline & enthesis & 40,5 & 44,1 & 1,2 & 261,9 \\
\hline \multirow[t]{5}{*}{ OP 2} & $\mathrm{Mtj}$ & 38,5 & 26,9 & 2,1 & 170 \\
\hline & body & 45,5 & 35,8 & 1,3 & 287 \\
\hline & pre insertional & 47,7 & 44,9 & 1,1 & 182,8 \\
\hline & enthesis & 60,1 & 51,6 & 3,2 & 305,5 \\
\hline & Sagittal & mean & SD & $\min$ & $\max$ \\
\hline \multirow[t]{4}{*}{ OP 1} & $\mathrm{Mtj}$ & 96,7 & 107,8 & 1,8 & 641,4 \\
\hline & body & 80,4 & 73,6 & 2 & 281 \\
\hline & pre insertional & 82,9 & 77,2 & 4,4 & 377,9 \\
\hline & enthesis & 53,8 & 41,2 & 3,9 & 207,6 \\
\hline \multirow[t]{6}{*}{ OP 2} & Mtj & 106,3 & 104,9 & 0,8 & 512 \\
\hline & body & 94,6 & 80,1 & 1,4 & 306,4 \\
\hline & pre insertional & 95,4 & 83,8 & 1,4 & 371,7 \\
\hline & enthesis & 67,0 & 76,9 & 1,7 & 475 \\
\hline & PLANTAR FLE & & & & \\
\hline & Axial & mean & SD & $\min$ & $\max$ \\
\hline \multirow[t]{4}{*}{ OP 1} & Mtj & 39,1 & 31,8 & 1 & 254,8 \\
\hline & body & 48,8 & 33,6 & 2,1 & 165 \\
\hline & pre insertional & 62,9 & 37,8 & 1,4 & 259,2 \\
\hline & enthesis & 66,5 & 51,1 & 1,9 & 281,8 \\
\hline
\end{tabular}


Continue from Table II.

\begin{tabular}{|c|c|c|c|c|c|}
\hline \multirow{5}{*}{ OP 2} & Mtj & 32,8 & 17,8 & 1,6 & 89,3 \\
\hline & body & 42,9 & 27,1 & 1,9 & 167,3 \\
\hline & pre insertional & 75,2 & 52,8 & 1,7 & 244 \\
\hline & enthesis & 61,2 & 57,3 & 0,6 & 258,4 \\
\hline & Sagittal & mean & SD & $\min$ & $\max$ \\
\hline \multirow[t]{4}{*}{ OP 1} & $\mathrm{Mtj}$ & 72,4 & 44,8 & 7,2 & 280,6 \\
\hline & body & 86,1 & 49,5 & 10,3 & 243,4 \\
\hline & pre insertional & 65,4 & 54,5 & 1,2 & 296,8 \\
\hline & enthesis & 62,3 & 46,8 & 3,7 & 197,2 \\
\hline \multirow[t]{4}{*}{ OP 2} & $\mathrm{Mtj}$ & 85,8 & 46,4 & 8,3 & 209,2 \\
\hline & body & 95,3 & 61,6 & 7,8 & 269,5 \\
\hline & pre insertional & 59,8 & 39,9 & 2,5 & 211,3 \\
\hline & enthesis & 73,4 & 58,7 & 1,4 & 241,3 \\
\hline
\end{tabular}

Table III. Inter observer reliability of SWE stiffness (KPA) of the 4 different portions of the tendon in axial and sagittal for both operators (op1: operator1; op2: operator2), in dorsal and plantar position. Mtj: Myo-tendinous junction.

\begin{tabular}{|c|c|c|c|c|c|}
\hline \multicolumn{4}{|c|}{ PLANTAR FLEXION } & \multicolumn{2}{|c|}{ DORSAL FLEXION } \\
\hline Axial & Mtj & 0.30526 & Axial & Mtj & 0.13377 \\
\hline & Body & 0.30449 & & Body & 0.095516 \\
\hline & Pre insertional & - & & Pre insertional & - \\
\hline & Enthesis & 0.27311 & & Enthesis & 0.16907 \\
\hline \multirow[t]{4}{*}{ Sagittal } & Mtj & 0.16376 & Sagittal & Mtj & - \\
\hline & Body & - & & Body & 0.23587 \\
\hline & Pre insertional & 0.15332 & & Pre insertional & 0.070272 \\
\hline & Enthesis & 0.075153 & & Enthesis & 0.095756 \\
\hline
\end{tabular}

operator measured the stiffness at $10^{\circ}$ of dorsi-flexion, in AT body, on a dynamometer in order to have a constant strength on the tendon with a mean stiffness of $83,2 \mathrm{kPa}$. Considering the 4 AT levels, we made the same statement and found no difference between both AT side. Considering AT body in plantar flexion, our values were respectively $45,9 \mathrm{kPa}$ and $90,7 \mathrm{kPa}$ in axial and sagittal position and are linkable with this SWE literature.

Then, in the most important cohort, Fu et al. measured SWE in AT body on 326 healthy volunteers in axial and sagittal in relaxed position of the ankle ${ }^{24}$. They showed a good interobserver reproducibility $(0,923$ for axial measurement and 0,870 for sagittal) in this plantar flexion, with no significant difference with age. Considering AT body in plantar flexion, our values also correlates with these previous data.

In our series, we divided AT in 4 portions based on
Del Buono et al. functional description. Del Buono et al. divided the tendon and have taken in consideration AT anatomic regions and then biomechanical function to class AT in non-insertional and insertional parts, with muscular fibers for the non-insertional and different management between these two main regions ${ }^{17}$. In literature and in our personal experience, this could be explained by the histologic structure of the tendon which is different between AT musculotendinous junction, body and insertional parts with different biomechanical functions. Physiologically, a tendon is formed by collagen fibers, associated with striated muscle cells in smaller quantities than in muscle in its myo-tendinous junction ${ }^{25}$. The healthy body and the pre-insertional tendon are principally composed of type 1 collagen and a few elastic fibers, within a ground substance containing cells (tenocytes and tenoblastes) and water. Concerning the enthesis, 


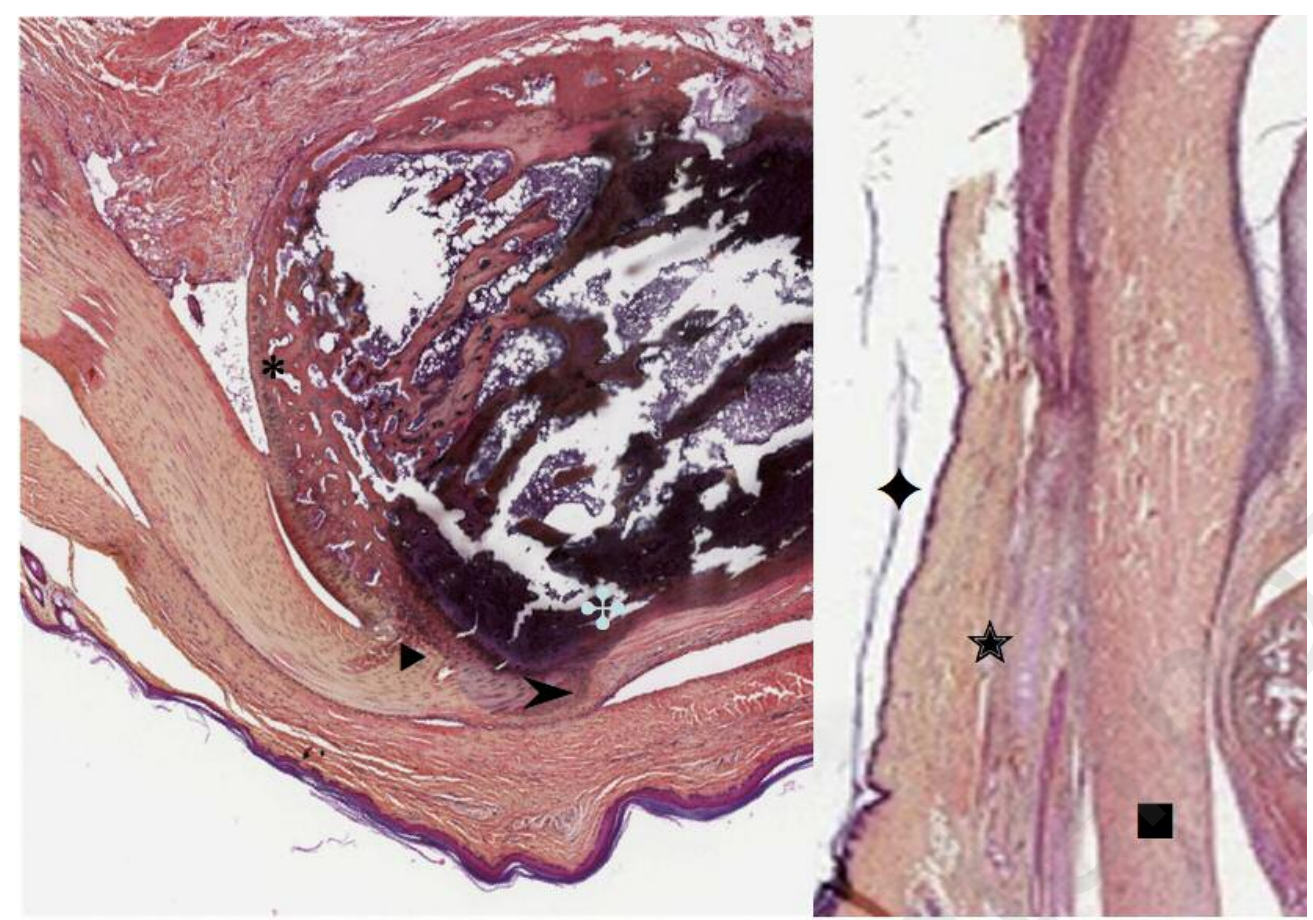

Figure 4. Sagittal histological section of a rat tendon showing the three insertional tendinous structures ( sesamoido-fibrocartilage, $>$ entheso-fibrocartilage and $\because$ periosto-fibrocartilage) and musculo-tendinous structures ( $\square$ body tendon, $\star$ muscle, $\uparrow$ skin).

three parts can be distinguished: sesamoido-fibrocartilage, entheso-fibrocartilage and periosto-fibrocartilage (Fig. 4) ${ }^{26}$. This could explain our results with a stiffer tendon near the enthesis.

On the other hand, a former qualitative SE study, by De Zordo et al., divided the tendon in 3 portions (1: musculo-tendinous junction; 2: 2-6 cm above insertion of the calcaneus and 3: the insertion of the calcaneus) founded also the same differences than ours concerning AT stiffness with $S^{11}$. In a more recent cadaverer's study, Haen et al. divided tendon in 3 portions and showed a significant difference in quantitative SWE values in function of the strain of the tendon but, unfortunately, they didn't analyze the difference between these 3 portions ${ }^{16}$. However, they focused, like in our own experience, about an increase of the AT stiffness in maximal dorsal flexion.

In the same way, a recent study of Payne et al. presented also contradictory results with SE elastography (on the tendon's body in one unique site) with poor reproducibility of compression elastography in AT, considering same day and consecutive day measurements ${ }^{27}$.

With SWE elastography, the same ward team found, in a very recent series, that reproducible SWE measures were obtained over a $1-\mathrm{h}$ period as well as a period of 5 consecutive days with more reliable measures obtained using a relaxed foot position ${ }^{28}$. These very recent results are concordant with ours. Indeed, our results are less reproducible concerning SWE measurements in the dorsal flexion, and the SD are much larger in this position. This should make us think that the most interesting value is the plantar flexion SWE measurement.

Our study has however 2 main limitations. First, this study was constrained by the small size of patients $(n=15)$ but to our knowledge, it is the largest homogeneous series, with a prospective design, focusing on AT healthy tendon quantitative SWE, not considering AT as a unique tube entity. Second, neither intra-observer agreement was calculated. However, our goal was to evaluate, in clinical practice, SWE quantitative assessment in AT and not intraobservater MSK radiologist reproducibility. Last, we didn't use any pad like previous studies, but we proceed like this to be as close as possible to the daily practice.

Our study is a great amount of data collected on few normal AT with 2 MSK operators in the same exact conditions of SWE measurements, considering its zonal anatomy and trying to explain SWE differences in correlation with biomechanical and histology aspects. According to these results, we propose a reliable and simplified AT US dynamic protocol, in a reasonable time, with a 2-time cluster: 1) Dorsal Flexion with anatomic measurements and tendinopathy assessment in B-mode - 2) Plantar Flexion with vascular and functional stiffness assessment in PowerDoppler and SWE mode. Indeed, the perspective of our study is to detect pre-morphological changes in very early or tenuous AT symptomatic tendinopathy, due to the SWE analysis, such as Dirrichs et al. considering SWE for the evaluation of patients with 
tendinopathies with increased sensitivity from 67.1 to $94.3 \%$ using SWE or for monitoring of treatment of these tendinopathies ${ }^{29,30}$.

\section{Conclusion}

Our study investigated the SWE quantitative variable stiffness value in all the 4 parts of AT, in correlation with its zonal anatomy. According to these results, we propose a reliable and simplified AT US protocol with a 2-times cluster: 1) Dorsal Flexion with anatomic measurements and tendinopathy assessment in Bmode - 2) Plantar Flexion with vascular and functional stiffness assessment in Power-Doppler and SWE mode, in a reasonable time. Further series should be leaded using a prospective design and a large included cohort to confirm our results.

\section{Conflict of interest}

No potential conflict of interest relevant to this article was reported.

\section{Ethics}

The Authors declare that this research was conducted following basic ethical aspects and international standards as required by the Journal and recently update in $^{31}$.

\section{References}

1. Asplund CA, Best TM. Achilles tendon disorders. BMJ. 2013;346:f1262.

2. Maffulli N, Sharma P, Luscombe KL. Achilles tendinopathy: aetiology and management. J R Soc Med. 2004 Oct;97(10): 472-476.

3. Khan K, Forster B, Robinson J, Cheong Y, Louis L, Maclean L, et al. Are ultrasound and magnetic resonance imaging of value in assessment of Achilles tendon disorders? A two year prospective study. Br J Sports Med. 2003 Apr;37(2):149-153.

4. Sarvazyan A, Hall TJ, Urban MW, Fatemi M, Aglyamov SR, Garra BS. An overview of elastography - an emerging branch of medical imaging. Curr Med Imaging Rev. 2011 Nov;7(4): 255-282.

5. Domenichini R, Pialat J-B, Podda A, Aubry S. Ultrasound elastography in tendon pathology: state of the art. Skeletal Radiol. 2017 Aug 1.

6. Sigrist RMS, Liau J, Kaffas AE, Chammas MC, Willmann JK Ultrasound Elastography: Review of Techniques and Clinical Applications. Theranostics. 2017 Mar 7;7(5):1303-1329.

7. Nightingale K, McAleavey S, Trahey G. Shear-wave generation using acoustic radiation force: in vivo and ex vivo results. Ultrasound Med Biol. 2003 Dec;29(12):1715-1723.

8. Dietrich CF, Bamber J, Berzigotti A, Bota S, Cantisani V, Castera L, et al. EFSUMB Guidelines and Recommendations on the Clinical Use of Liver Ultrasound Elastography, Update 2017 (Long Version). Ultraschall Med Stuttg Ger 1980. 2017 Aug;38(4):e16-47.
9. Athanasiou A, Tardivon A, Tanter M, Sigal-Zafrani B, Bercoff J, Deffieux $T$, et al. Breast lesions: quantitative elastography with supersonic shear imaging-preliminary results. Radiology. 2010 Jul;256(1):297-303.

10. Fouré A. New Imaging Methods for Non-invasive Assessment of Mechanical, Structural, and Biochemical Properties of Human Achilles Tendon: A Mini Review. Front Physiol. $2016 \mathrm{Jul}$ 27. Available from: http://www.ncbi.nlm.nih.gov/pmc/articles/PMC4961896/

11. De Zordo T, Chhem R, Smekal V, Feuchtner G, Reindl M, Fink $\mathrm{C}$, et al. Real-time sonoelastography: findings in patients with symptomatic achilles tendons and comparison to healthy volunteers. Ultraschall Med Stuttg Ger 1980. 2010 Aug;31(4): 394-400.

12. Aubry S, Risson J-R, Barbier-Brion B, Tatu L, Vidal C, Kastler B. Transient elastography of calcaneal tendon: preliminary results and future prospects. J Radiol. 2011 May;92(5):421-427.

13. Siu W-L, Chan C-H, Lam C-H, Lee C-M, Ying M. Sonographic evaluation of the effect of long-term exercise on Achilles tendon stiffness using shear wave elastography. J Sci Med Sport. 2016 Nov; $19(11): 883-887$.

14. Capalbo E, Peli M, Stradiotti P. Sonoelastography of the distal third of the Achilles tendon in asymptomatic volunteers: correlation with anthropometric data, ultrasound findings and reproducibility of the method. Radiol Med (Torino). 2016 Aug;121 (8):667-674.

15. Aubry S, Nueffer J-P, Tanter M, Becce F, Vidal C, Michel F. Viscostiffness in Achilles tendonopathy: quantitative assessment by using real-time shear-wave elastography. Radiology. 2015 Mar;274(3):821-829.

16. Haen TX, Roux A, Soubeyrand M, Laporte S. Shear waves elastography for assessment of human Achilles tendon's biomechanical properties: an experimental study. J Mech Behav Biomed Mater. 2017 May;69:178-184.

17. Del Buono A, Chan O, Maffulli N. Achilles tendon: functional anatomy and novel emerging models of imaging classification. Int Orthop. 2013 Apr;37(4):715-721.

18. Abate M, Silbernagel KG, Siljeholm C, Di lorio A, De Amicis D, Salini $V$, et al. Pathogenesis of tendinopathies: inflammation or degeneration? Arthritis Res Ther. 2009;11(3):235.

19. Holmes GB, Lin J. Etiologic factors associated with symptomatic achilles tendinopathy. Foot Ankle Int. 2006 Nov;27 (11):952-959.

20. Kaux J-F, Delvaux F, Oppong-Kyei J, Dardenne N, Beaudart $C$, Buckinx $F$, et al. Validity and reliability of the French translation of the VISA-A questionnaire for Achilles tendinopathy. Disabil Rehabil. 2016 Dec;38(26):2593-2599.

21. Jhingan S, Perry M, O'Driscoll G, Lewin C, Teatino R, Malliaras $P$, et al. Thicker Achilles tendons are a risk factor to develop Achilles tendinopathy in elite professional soccer players. Muscles Ligaments Tendons J. 2011 Dec 29;1(2):5156.

22. Fujihara Y, Matsumura T, Murayama N, Motoki M, Mitake T. Development of acoustic coupler for elastography. Medix $2011 ; 55: 40-44$. !

23. Suydam SM, Soulas EM, Elliott DM, Silbernagel KG, Buchanan TS, Cortes DH. Viscoelastic properties of healthy achilles tendon are independent of isometric plantar flexion strength and cross-sectional area. J Orthop Res Off Publ Orthop Res Soc. 2015 Jun;33(6):926-931.

24. Fu S, Cui L, He X, Sun Y. Elastic Characteristics of the Normal Achilles Tendon Assessed by Virtual Touch Imaging Quantification Shear Wave Elastography. J Ultrasound Med Off J Am Inst Ultrasound Med. 2016 Sep;35(9):1881-1887.

25. Kirchgesner T, Larbi A, Omoumi P, Malghem J, Zamali N, Manelfe J, et al. Drug-induced tendinopathy: from physiology to clinical applications. Jt Bone Spine Rev Rhum. 2014 Dec;81(6):485-492. 
26. Han M, Larson PEZ, Liu J, Krug R. Depiction of achilles tendon microstructure in vivo using high-resolution 3-dimensional ultrashort echo-time magnetic resonance imaging at $7 \mathrm{~T}$. Invest Radiol. 2014 May;49(5):339-345.

27. Payne C, Webborn N, Watt P, Cercignani M. Poor reproducibility of compression elastography in the Achilles tendon: same day and consecutive day measurements. Skeletal Radiol. 2017 Jul;46(7):889-895.

28. Payne C, Watt P, Cercignani M, Webborn N. Reproducibility of shear wave elastography measures of the Achilles tendon. Skeletal Radiol. 2017 Dec 19.
29. Dirrichs T, Quack V, Gatz M, Tingart M, Kuhl CK, Schrading S. Shear Wave Elastography (SWE) for the Evaluation of Patients with Tendinopathies. Acad Radiol. 2016 Oct;23(10): 1204-1213

30. Dirrichs T, Quack V, Gatz M, Tingart M, Rath B, Betsch M, Kuhl CK, Schrading S. Shear Wave Elastography (SWE) for Monitoring of Treatment of Tendinopathies: A Double-blinded, Longitudinal Clinical Study. Acad Radiol. 2017 Nov 16.

31. Padulo J, Oliva F, Frizziero A, Maffulli N. Muscles, Ligaments and Tendons Journal - Basic principles and recommendations in clinical and field science research: 2016 update. MLTJ. 2016;6(1):1-5. 